\title{
Conformación de Lotes Mínimo Tiempo en la Operación de Acomodo Considerando $k$ Equipos Homogéneos usando Metaheurísticos
}

\author{
Rodrigo A. Gómez ${ }^{(1,2)}$, Oscar G. Giraldo ${ }^{(2)}$ y Emiro A. Campo ${ }^{(1)}$ \\ (1) Grupo de investigación en logística y Administración de cadena de suministro (GILACS), Escuela \\ Superior en Administración de la Cadena de Suministro (ESACS), Institución Universitaria Esumer, Cra. 87 \\ \#30-65, Medellín - Colombia. \\ (2) Grupo de Investigación Escuela de Prospectiva y Desarrollo Empresarial, Institución Universitaria \\ Esumer, Maestría en Gestión de Organizaciones con Univ. de QUEBEC (Canadá) y EAN-ESUMER \\ (Colombia), Cl. 76 \#80126, Medellín - Colombia. \\ (e-mail: ragomez@elpoli.edu.co; ogiraldo@esumer.edu.co; ecampo@udem.com)
}

Recibido Mar. 11, 2016; Aceptado Abr. 27, 2016; Versión final Jun. 12, 2016, Publicado Dic. 2016

\begin{abstract}
Resumen
Se desarrolla y valida un metaheurístico para la conformación de lotes de acomodo del mínimo tiempo posible considerando $k$ equipos de manejo de materiales homogéneos. El método debe servir también para la ubicación del depósito (depot, punto de inicio y finalización de la preparación de pedidos), y para determinar el tamaño de la lista de productos a acomodar, entre otros factores. La validación experimental fue realizada usando diseño estadístico de parcelas dividas. Como resultado de la experimentación se obtuvo que el metaheurístico denominado Búsqueda Inteligente en la Vecindad (INS) produjo ahorros de tiempo entre 24 y $50 \mathrm{~min} /$ lote respecto a la regla "el Primero que llega es el primero en ser servido (FCFS). Se muestra que el metaheurístico propuesto no solo contribuye al avance en el tema logístico sino también a la eficiencia operacional de la gestión de almacenes.
\end{abstract}

Palabras clave: acomodo, almacén, conformación de lotes, logística, metaheurístico.

\section{Minimum Time Order Batching in the Put away Operation Considering k Homogeneous Equipment using metaheuristics}

\begin{abstract}
This paper presents the development and validation of a metaheuristic for minimum time order batching in put away operations considering $\mathrm{k}$ material handling equipment. The method also serves to locate the depot and to determine the put away list size, among other factors. Experimental validation was performed by a statistical design named split plot. As a result of the experiment it was obtained that metaheuristic INS (Intelligent Neighborhood Search) generated time savings between 24 and 50 minutes per batch compared with the classical rule FCFS (First Come, First Served). It is demonstrated that the proposed metaheuristic not only contributes to logistic but also to the operational efficiency of warehouse management.
\end{abstract}

Keywords: put away, warehouse order batching, logistics and metaheuristic. 


\section{INTRODUCCIÓN}

El acomodo es una operación del CEDI, que permite trasladar los productos desde el muelle de descargue o de recepción a las posiciones de almacenamiento asignadas utilizando equipos de manejo de materiales o personal, según las características de los productos que se manipulan y la disponibilidad de recursos en el proceso logístico (Frazelle y Sojo, 2006; Van der Berg, 2007). Para Bartholdi y Hackman (2014), el acomodo representa aproximadamente el $15 \%$ de los costos en CEDI no automatizados, ya que en este tipo de almacenes, se suelen requerir cantidades considerables de personal para ubicar los productos en las posiciones de almacenamiento asignadas. Adicionalmente, el acomodo, puede afectar la eficiencia de la preparación de pedidos, ya que esta operación, se encarga de recoger y organizar los productos almacenados, con el fin de atender los requerimientos de los clientes en las condiciones logísticas pactadas (Bartholdi y Hackman, 2014; Theys, Bräysy, Dullaert y Raa, 2010; Yu y Koster, 2010).

En la gestión del acomodo, se consideran decisiones de: a) asignación de posiciones de almacenamiento (slotting) y b) conformación de lotes con ruteo para ubicar los productos en las posiciones de almacenamiento, empleando equipos de manejo de materiales o personal (Van der Berg, 2007; Bartholdi y Hackman, 2011; Henn; 2012). A partir de los resultados de una revisión del estado del arte sólo se identificaron modelos de optimización (Takahama et al., 2002; Heragu et al., 2005; Hou et al.2010; Kutzelnigg, 2011) y metaheurísticos (Muppani y Adil, 2008; Kim y Smith, 2012; Pazour y Carlo, 2015) que apoyan decisiones de slotting o asignación de posiciones de almacenamiento. Debe resaltarse que el slotting no considera la conformación de lotes y ruteo para acomodar los productos en las posiciones asignadas buscando obtener tiempos mínimos que impacten en la eficiencia y la eficacia de la operación. Para superar ese vacío, se estableció como objetivo modelar la conformación de lotes con ruteo en el acomodo considerando una flota de $\mathrm{K}$ equipos homogéneos que se soluciona con un metaheurístico con el fin de obtener el menor tiempo total posible para la operación. Con este enfoque, no sólo se busca contribuir a la eficiencia y eficacia del acomodo, sino también a la generación de conocimiento.

En este trabajo, el problema de conformación de lotes en el acomodo considerando una flota de $K$ equipos homogéneos es formulado para un almacén de productos terminados que tiene una layout con un pasillo central y cinco paralelos. Cada pasillo paralelo está conformado por un sistema de estanterías selectiva de profundidad simple de cuatro niveles de altura. En cada posición de almacenamiento se almacenan estibas con cajas de cartón que contienen los productos que permiten atender los requerimientos de los clientes y son manipulados logísticamente con operaciones de recepción, acomodo, almacenamiento, preparación de pedidos y despacho. Las rutas para acomodar o preparar los pedidos de los clientes inician y terminan en un punto físico del almacenen denominado depot.

El acomodo consiste en ubicar los productos que se reciben desde los proveedores en las posiciones de almacenamiento asignadas a través del método de slotting empleado por la empresa. El método de slotting implementa una estrategia de zonificación $A B C$ debido a rotación, cercanía a la zona de recepción y despacho así como restricciones según la naturaleza de carga del producto. Un lote de acomodo es una combinación de productos que deben ser ubicados en una posición de almacenamiento en el menor tiempo posible. Un lote debe cumplir con restricciones asociadas a la naturaleza de los productos, la capacidad de carga del equipo de manejo de materiales así como su capacidad para acceder a la posición de almacenamiento asignada a través de movimientos horizontales y verticales (altura). Para manipular los productos a acomodar se utiliza una flota de equipos de manejo de materiales homogéneos conformado por transpaletas manuales y eléctricas así como un order picker que alcanza un máximo de 5 metros de altura. Debe resaltarse que la flota de equipos se considera homogénea porque estos tienen las mismas velocidades, capacidad de carga y ejecución de movimientos en altura. El layout para el almacén de producto terminado en modelamiento se muestra en la figura 1.

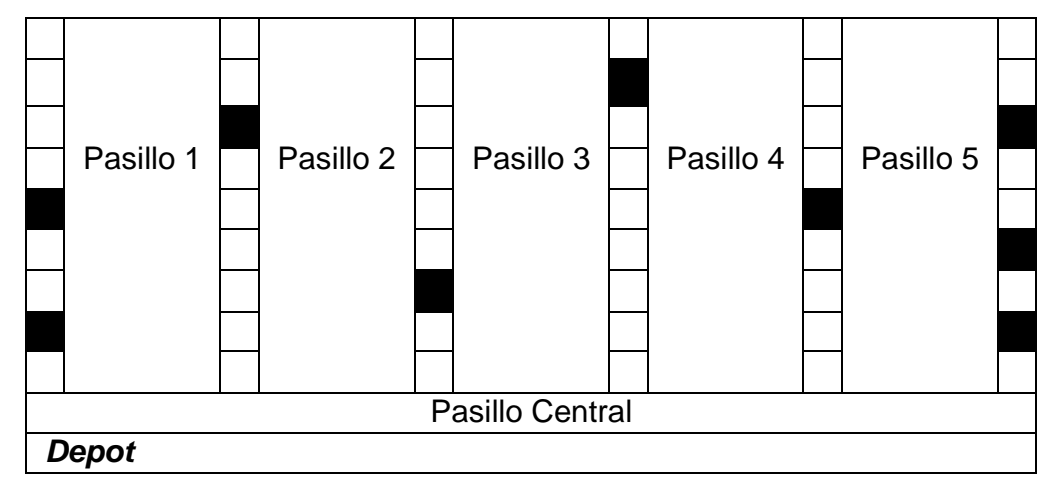

Fig. 1: Layout almacén de producto terminado en estudio 


\section{FORMULACIÓN DEL PROBLEMA}

En este apartado se formula el problema de conformación de lotes en el acomodo (Kulak et al., 2012; Henn y Schmid, 2013; Prins 2009 y Leung et al., 2013). El modelo se compone de una función objetivo, conjunta de parámetros y variables de decisión. La notación del modelo es descrita en lo que sigue separados en (1) indices y conjuntos; (2) parámetros; y (3) variables de decisión.

\section{Indices y conjuntos}

IEL Lote de acomodo (LA) I perteneciente a un conjunto de solución de lotes (CSLA) L.

$e, f \in D \quad$ Posiciones de almacenamiento.

$g \in D \quad$ Subconjunto de posiciones de almacenamiento.

$k \in K \quad$ EMMateriales (Equipos de Manejo de Materiales) disponibles en el CEDI.

$w \in W \quad$ Una ruta de acomodo $\left(\mathrm{s}^{\prime}\right) w$ del conjunto de rutas (CRS)W.

$i \in I \quad$ Producto $i$ perteneciente al total $/$ de las ordenes de recepción o lista de acomodo.

\section{Parámetros:}

$d^{\text {et }} \quad$ Distancia entre las posiciones de almacenamiento e y $f$.

$b_{i}^{\prime} \quad$ Cantidad de producto $i$ a ser recogido en un lote $L A^{\prime} \in C S L A_{L}$.

$L C_{i} \quad$ Capacidad de carga usado por un producto $i \in I$.

$V^{\prime} \quad$ Velocidad de viaje $(\mathrm{m} / \mathrm{s})$ de un EMMateriales $k \in K$ asignado a un LA' $\mathrm{CSLA}_{L}$

tbu' Tiempo de ubicación y manipulación de un producto $i \in L A^{\prime}$ utilizando un EMMateriales $k \in K$.

$\mathrm{ALC}_{\mathrm{k}} \quad$ Capacidad de carga disponible de un EMMateriales $k \in K$.

Variables de decisión

$$
S_{e f}^{l}=\left\{\begin{array}{l}
1, \text { Si un posición e es visitada inmediatamente después de una posición } f \text { en una ruta } \\
\text { asociada a un lote } L A^{\prime} \in \operatorname{CSLA} A_{L} \\
0, \text { De otro modo }
\end{array}\right.
$$

$X_{i}^{l}=\left\{\begin{array}{l}1, \text { Si un producto i está asignado a un } \operatorname{LA}^{\prime} \in \mathrm{CSLA}_{L} \\ 0, \text { De otro modo }\end{array}\right.$

$Y_{k}{ }^{l}=\left\{1\right.$, Si un EMMateriales $k$ es asignado a una ruta asociada a un $L A^{\prime} \in C S L A_{L}$

$N_{f}^{l}=\left\{\begin{array}{l}0, \text { De otro modo } \\ \begin{array}{l}1, \text { Si una posición } f \text { es visitada dentro de una ruta asociada a un } L A^{\prime} \in \operatorname{CSL} L A_{L} \\ 0, \text { De otro modo }\end{array}\end{array}\right.$

Formulación del modelo:

$\operatorname{Min} T=\sum_{l \in L} \sum_{k \in K} \sum_{e \neq f \in D} \frac{d^{l}{ }_{e f}}{V^{l}} \cdot Y_{k}{ }^{l}++\sum_{l \in L} \sum_{i \in I} b_{i}^{l} \cdot t b u^{l} \cdot X_{i}^{l}$

Sujeto a:

$\sum_{l \in L} X_{i}^{l}=1 \quad \forall l \in L$

$\sum_{i \in I} b^{l}{ }_{i} L C_{i} X^{l}{ }_{i} \leq A L C_{k} Y_{k}{ }^{l} \quad \forall l \in L, k \in K$

$\sum_{f \in S, f \neq e} S_{e f}^{l}=N^{l} e^{\forall} l \in l, e \in D$

$\sum_{e \in S, f \neq e} S_{e f}^{l}=N_{f}^{l} \forall l \in L, f \in D$

$\sum_{e \in g, e \in D \backslash g} S_{e f}^{l} \geq N_{f}^{l} \forall l \in L, f \subset D$

$d_{e f}^{l}, X_{i}^{l}, Y_{k}^{l}, N_{e}^{l} \in(0,1)$ 
La función objetivo (1) minimiza el tiempo total para el conjunto de lotes con ruteo. La restricción (2) asegura que se asigna cada producto i a un $L A \in C S L A_{L}$. La restricción (3) asegura que la capacidad de carga de un EMMateriales (Equipos de Manejo de Materiales) $k \in K$ no es excedida por la cantidad de productos $i$ asignados al $L A^{\prime} \in C S L A_{L}$. Las restricciones (4) y (5) aseguran que cada posición de almacenamiento de una ruta asociada a un lote $l \in L$ tiene un predecesor y un sucesor. La restricción (6) evita subtours en el conjunto de $g$ posiciones de almacenamiento. Finalmente, la restricción (7) garantiza que las variables de decisión sean positivas.

\section{REGLA DE PRIORIDAD Y METAHEURÍSTICO}

En esta sección son modeladas una regla de prioridad FCFS (First Come First Served) y un metaheurístico denominado INS (Intelligent Neighborhood Search) para resolver el problema de conformación de lotes en el acomodo considerando $K$ equipos homogéneos.

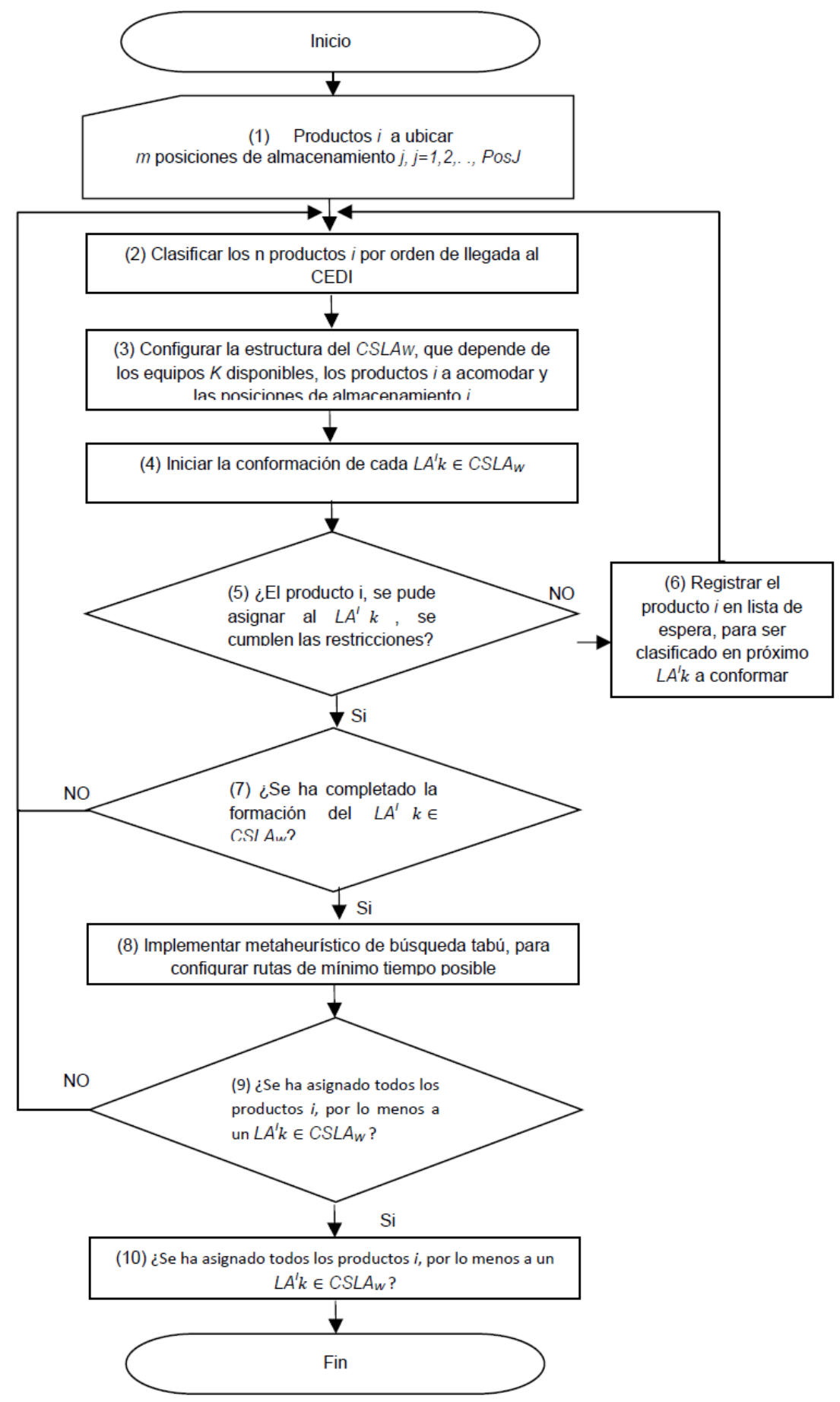

Fig. 2. Regla de prioridad FCFS para la conformación de lotes 
La regla FCFS para la generación de lotes de acomodo. La regla FCFS, permite formar conjuntos de $L A^{\prime}$ denominados CSLA $L$ asignando los productos $i$ por orden de llegada en la ventana del tiempo programada hasta completar la capacidad de los equipos EMMateriales $k \in K$ programados. A cada $L A^{\prime} \in C S L A_{L}$, se le implementa un metaheurístico de búsqueda tabú para obtener una ruta con $m$ posiciones de almacenamiento $j$ que se deben visitar para acomodar los $n$ productos $i$, en el menor tiempo posible.

Las etapas descritas de conformación de lotes con ruteo, en el acomodo con la regla FCFS (Fig.2), se implementan hasta asignar cada uno de los $n$ productos $i$ a un $L A^{\prime} \in C^{\prime} S L A_{L}$. La gestión de la información de la conformación de lotes con ruteo, los productos $i$, las posiciones de almacenamiento $j$, los EMMmateriales $K$ asignados, se realiza en el WMS en tiempo real.

\section{Metaheurístico INS para la formación de lotes}

En este apartado es desarrollado el metaheurístico INS, el cual tiene como objetivo formar conjuntos de lotes de acomodo CSLA $L$ del menor tiempo posible. Este metaheurístico INS es un enfoque novedoso no detectado en la literatura que estudia los movimientos entre y dentro de lotes, para formar nuevas soluciones. A continuación, se presentan las etapas del metaheurístico INS.

\section{Etapa 1: Clasificar EMMateriales}

Se clasifica cuáles de los EMMateriales homogéneos $k \in K$ pueden acomodar los $n$ productos en las $m$ posiciones de almacenamiento asignadas. Se diseña un procedimiento en el WMS, que permite clasificar cuales productos $i$ pueden ser acomodados por cada EMMateriales $k \in K$ en las posiciones de almacenamiento $j$ asignadas. Esta condición es una de las más importantes en el problema, ya que un EMM $k \in K$ puede ejecutar el acomodo dependiendo de las características de los $n$ productos a ubicar y el nivel de altura de las posiciones de almacenamiento a visitar. La estructura de esta clasificación se presenta en la Tabla 1.

Tabla 1: Clasificación de EMMateriales $K$, productos $i$ y posiciones de almacenamiento $j$.

\begin{tabular}{|c|c|c|}
\hline Producto $i$ & Posición $j$ & EMM K \\
\hline Producto 1 & Pos 1 & EM1 \\
\cline { 2 - 3 } & $\ldots \ldots \ldots$ & $\ldots \ldots \ldots$ \\
\cline { 2 - 3 } & Pos $j$ & EMk \\
\hline Producto 2 & Pos 3 & EM1 \\
\cline { 2 - 3 } & $\ldots \ldots \ldots$ & \\
\cline { 2 - 3 } & Pos $j$ & EMk \\
\hline$\ldots \ldots \ldots \ldots \ldots$ & $\ldots \ldots \ldots \ldots$ \\
\hline Producto $i$ & Pos $j$ & $\ldots \ldots \ldots \ldots$ \\
\hline
\end{tabular}

Etapa 2: Generar un conjunto inicial de lotes de acomodo CSL $A_{\text {Linicial }}$

El conjunto de lotes inicial $C S L A_{\text {Linicial }}$ es generado con la implementación de la regla de prioridad FCFS descrita arriba. Para conformar el conjunto inicial, se emplea la información de la clasificación de los EMMateriales $k \in K$, productos $i$ y las posiciones de almacenamiento $j$ desarrollado en la etapa 1 de la presente sección. La formación del $C S L A_{\text {Linicial }}$ está basada en una estructura, que contiene $L A^{\prime}$ lotes de acomodo, los $n$ productos $i$ a ubicar y $k \in K$ EMMateriales disponibles (Tabla 2). Además, el número de $L A^{\prime} \in C S L A_{\text {Linicial }}$ depende de la cantidad de productos a ubicar o los EMMateriales etiquetados como programables en el CEDI así como las restricciones de estos asociados con las capacidades Cd (capacidad disponible) y $\mathrm{Cu}$ (capacidad utilizada).

Tabla 2: Estructura de $L A^{\prime} \in C S L A L_{\text {inicial }}$

\begin{tabular}{|c|c|c|c|c|c|c|c|c|}
\hline \multicolumn{2}{|c|}{ Lotes $L A^{\prime}$ y EMMk } & Producto 1 & Producto 2 & Producto 3 & $\ldots$ & Producto $i$ & $\mathrm{Cd}$ & $\mathrm{Cu}$ \\
\hline$L A^{1}$ & $E M M k$ & $P 11$ & $P 12$ & $P 13$ & $\ldots$ & $P 1 i$ & $\mathrm{Cd}_{1}$ & $\mathrm{Cu}_{1}$ \\
\hline$L A^{2}$ & $E M M k$ & $P 21$ & $P 22$ & $P 23$ & $\ldots$ & $P 2 i$ & $\mathrm{Cd}_{2}$ & $\mathrm{Cu}_{2}$ \\
\hline$\ldots \ldots$. & $\ldots \ldots$. & $\ldots \ldots$. & $\ldots \ldots$ & $\ldots \ldots$ & $\ldots$ & $\ldots \ldots$. & $\ldots \ldots$ & $\ldots$. \\
\hline$L A^{\prime}$ & $E M M k$ & $P 11$ & $P 12$ & $P 13$ & $\ldots$ & $P l i$ & $\mathrm{Cd}_{1}$ & $\mathrm{Cu}_{1}$ \\
\hline
\end{tabular}


Una vez que la estructura es diseñada, se comienza la conformación de los $L A^{\prime} \in C S L A_{\text {Linicial }}$ Los productos son asignados a cada $L A^{\prime} \epsilon C S L A_{\text {Linicial }}$ en el orden establecido por la regla FCFS (etapa 1). La conformación de un lote es completado cuando su capacidad de carga disponible es asignada y se verifica el cumplimiento de sus restricciones. Por esta razón, cada vez que un producto es asignado a un $L A^{\prime} \epsilon$ $C S L A_{\text {Linicial, }}$, e evalúa si la capacidad de carga utilizada es menor o igual a la disponible. Adicionalmente, se comprueba que un producto $i$ pueda ser programado en el lote según las características del EMMateriales $k \in K$ y la posición de almacenamiento $j$ asignada. Una vez, se forman cada uno de los $L A^{\prime} \in C S L A_{L \text { inicial }}$ se les implementa una de las variantes del metaheurístico de búsqueda tabú, con el fin de configurar rutas de mínimo tiempo total para la operación. A continuación, se representa la estructura que relaciona los lotes con ruteo en el acomodo, en el cual $\mathrm{P}_{i}^{\prime}$ indica que el producto $i$ es asignado al lote $L A^{\prime}$ y su rutas ${ }_{w}^{\prime}$

Tabla 3: Estructura conformación de lotes con ruteo, en el acomodo

\begin{tabular}{|c|c|c|c|c|c|c|}
\hline \multicolumn{6}{|c|}{$L A^{\prime} \in \mathrm{CSLA}_{L}$} & \multirow{3}{*}{$\begin{array}{l}\text { Tiempo de la ruta asociada a un } L A^{\prime} \\
\qquad s^{1}=t L A^{1}\end{array}$} \\
\hline \multirow{2}{*}{$\mathrm{LA}^{1}$} & \multirow{2}{*}{ EMk } & Productos & $\mathrm{P}^{1} 1$ & $P^{1} 2$ & & \\
\hline & & Ruta $s^{1}{ }_{w}$ & Pos1 & Pos 8 & & \\
\hline \multirow{2}{*}{$\mathrm{LA}^{2}$} & \multirow{2}{*}{ EMk } & Productos & & & $P^{2} 3$ & \multirow[b]{2}{*}{$s^{2}=t L A^{2}$} \\
\hline & & Ruta $s^{2}{ }_{w}$ & & & Pos 9 & \\
\hline$\ldots .$. & ........ & & $\ldots \ldots$. & $\ldots \ldots$. & $\ldots \ldots$. & $\ldots \ldots \ldots$ \\
\hline \multirow[t]{2}{*}{$\mathrm{LA}^{\prime}$} & \multirow[t]{2}{*}{ EMk } & Productos & $P^{\prime} 1$ & $P^{\prime} 2$ & $P_{i}^{\prime}$ & \multirow[t]{2}{*}{$s^{\prime}=t L A^{\prime}$} \\
\hline & & Ruta $s_{w}^{\prime}$ & Posj & Pos $j$ & Posj & \\
\hline
\end{tabular}

Una vez que se calculan los tiempos de las rutas de acomodo (tLA') integrados a $L A^{\prime} \in C S L A_{L}$, estos se suman para medir el tiempo el tiempo total de la operación utilizando la siguiente ecuación:

$T_{\text {Ltotalinicial }}=\sum_{l=1}^{l} \sum_{k=1}^{k} t L A^{l}$

$\mathrm{T}_{\text {Ltotalinicial }}$ es usado como solución semilla en la primera iteración del metaheurístico INS. De esta manera, se demuestra que la conformación de lotes con ruteo, se modelan de una forma integrada.

Etapa 3: Generar nuevos conjuntos de lotes CSLA $A_{\text {Lnuevo }}$ y movimientos en el metaheurístico INS.

Para formar nuevos $L A^{\prime} \epsilon C S L A_{L n u e v o}$, se implementan movimientos factibles de productos $i$ y posiciones de almacenamiento $j$ entre y dentro de lotes $L A^{\prime} \epsilon C S L A_{L a c t u a l}$ (inicial), los cuales son creados por el metaheurístico INS. A continuación, se describen las características de los dos grupos de movimientos, que se ejecutan en el metaheurístico INS de manera secuencial e integrada.
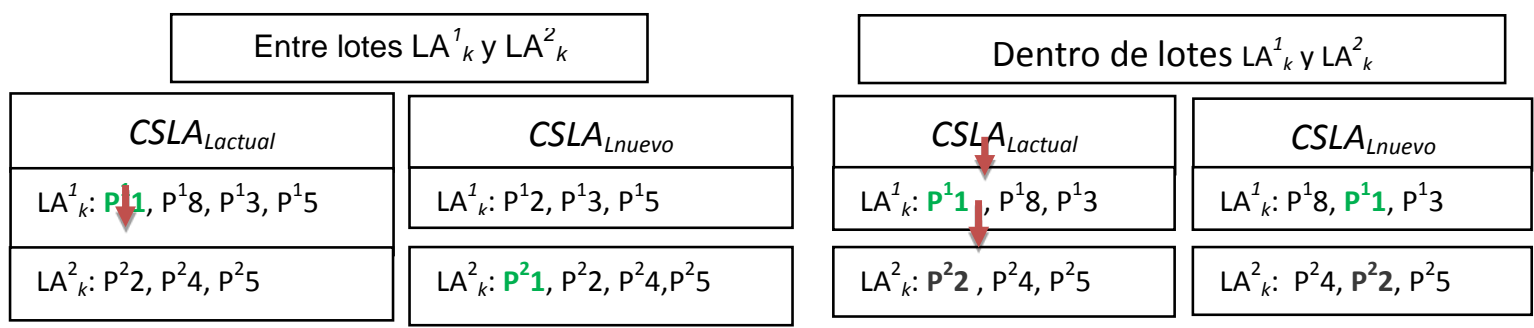

Fig.3.Movimientos de productos $i$ entre y dentro $L A^{\prime}$ para generar un $C S L A_{L \text { nuevo }}$

Grupo 1: Movimientos de inserción de productos $i$ entre y dentro de $L A^{\prime} \epsilon C S L A_{L}$ (e manera secuencial e integrada). El movimiento del producto $i$ a implementar, se elige al azar con método de selección programado en el metaheurístico INS.

Grupo 2: Una vez, se forman los nuevos lotes $L A^{\prime} \epsilon \operatorname{CSLA}_{L n u e v a}$ con el grupo de movimientos uno, se implementa el grupo de movimientos dos, que consiste en intercambiar posiciones de almacenamiento $j$

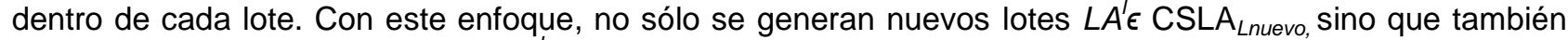
se obtienen sus rutas integradas $s_{W} \in C R S w$, las cuales buscan acomodar los $n$ productos $i$, en las $m$ posiciones de almacenamiento $j$ asignadas, en el menor tiempo posible. Por lo tanto, para ejecutar el grupo de movimientos dos, se implementa uno de los metaheurísticos de búsqueda tabú para resolver el componente de ruteo integrado a la conformación de lotes. 
Luego de medir los tiempos de las rutas $\left(t L A^{\prime}\right) s_{w}^{\prime} \epsilon L A_{\text {nueva, }}^{\prime}$ se calcula el tiempo total delCSLA $A_{L n u e v o}$ por medio de la siguiente ecuación:

$T_{\text {Ltotalnuevo }}=\sum_{r=1}^{R} \sum_{k=1}^{K} t L A^{l}$

Debe tenerse en cuenta, que para medir el tiempo de acomodo $\left(t L A^{\prime}\right)$ de cada $L A^{\prime} \epsilon \mathrm{CSLA}_{L n u e v o}$, se utiliza el grupo de funciones objetivo del metaheurístico de ruteo seleccionado para resolver este componente, los cuales se modelan en la sección. Una vez los $L A^{\prime} \epsilon C S L A_{L n u e v o}$ son conformados, las siguientes condiciones deben ser evaluadas: (1) La capacidad utilizada (cu) de un EMMateriales $\mathrm{k} \in K$ asignado a un $L A{ }^{\prime} \epsilon$ $C S L A_{L n u e v o}$ es menor o igual que su capacidad de carga disponible (cd); y (2) El EMMateriales $\mathrm{k} \in K$ programado a un $L A^{\prime} \epsilon C S L A_{L n u e v o}$ debe tener la capacidad de ejecutar movimientos horizontales y verticales para acomodar los $n$ productos $i$ en las $m$ posiciones de almacenamiento $j$. Cada unidad del producto $i$ es asignado únicamente a un a $L A^{\prime} \in C S L A_{L n u e v o}$ para evitar duplicaciones y soluciones no factibles.

Etapa 4: Analizar si $T_{\text {Lnuevototal }} \in C S L A_{L \text { nuevo }}$ es mejor que $T_{L m e j o r} \in C S L A_{L m e j o r}$

Si $T_{\text {Lnuevototal }} \in \mathrm{CSLA}_{L \text { nuevo }}$ es menor que $\mathrm{T}_{\text {Lmejor }} \epsilon \mathrm{CSLA}_{L \mathrm{Lmejor}}$ entonces el conjunto $C S L \mathrm{~A}_{L \text { nuevo }}$ es establecido como la mejor solución para el problema de conformación de lotes con ruteo $\left(C S L A_{L n u e v o}=C S L A_{L m e j o r}\right)$. En la primera iteración del metaheurístico INS $\mathrm{T}_{\text {Ltotalinicial }} \in \mathrm{CSLA}_{\text {Linicial }}$ corresponde a $\mathrm{T}_{L \text { mejor }} \epsilon C S L A_{L m e j o r}$. La evaluación descrita en esta etapa es realizada en cada iteración del metaheurístico INS.

\section{Etapa 5: Evaluar las condiciones de terminación}

El metaheurístico INS finaliza su ejecución hasta que alguno de los siguientes criterios de terminación se cumple. Los criterios establecidos son: i) el límite de iteraciones es alcanzado, ii) todos los movimientos entre y dentro de los lotes $L A^{\prime} \epsilon C S L A_{L a c t u a l}$ son ejecutados o iii) el tiempo de procesamiento computacional es excedido.

\section{RESULTADOS Y DISCUSIÓN}

La pregunta de investigación que soporta la experimentación estadística a realizar es la siguiente: ¿Evaluar, las diferencias de tiempo y eficiencia que produce metaheurístico INS integrado al WMS respecto a la regla de prioridad para resolver el componente de conformación de lotes en la operación de acomodo?. La Tabla 4 presenta las variables y factores modelados para la experimentación.

Para el diseñar las rutas de los conjuntos de lotes de acomodo conformados se implementa un metaheurístico de búsqueda tabú, el cual fue programado en JAVA $\AA$ utilizando el framework OpenTS ${ }^{\circledR}$. Para el metaheurístico de búsqueda tabú para el ruteo se considera una Ternure List de 7 , se establece un número de 1000 iteraciones y se implementan movimientos simples de intercambio e inserción para generar las nuevas rutas. Este metaheurístico fue validado utilizando 2000 corridas de prueba que demostraron su robustez para solucionar el problema de ruteo. Un diseño de experimentos denominado parcelas divididas es seleccionado para desarrollar el modelamiento y análisis estadístico de los resultados. Este diseño es seleccionado porque el factor grupo de K EMMateriales es difícil de cambiar en la experimentación. Esto se plantea porque se deben utilizar los mismos grupos de K EMMateriales en las 48 corridas ejecutadas durante las dos replicas diseñadas

Tabla 4 Variables y factores para la experimentación en la conformación de lotes

\begin{tabular}{|c|c|}
\hline Variable de respuesta & Tiempo promedio para el acomodo de los lotes conformados (minutos) \\
\hline Factor & Niveles \\
\hline \multirow{2}{*}{$\begin{array}{l}\text { Estrategia de conformación de } \\
\text { lotes (ECL) (F1). }\end{array}$} & n1: Regla de prioridad FCFS \\
\hline & n2:Metaheurístico NS basado en búsqueda en vecindarios \\
\hline \multirow{2}{*}{$\begin{array}{l}\text { Lista del tamaño de acomodo } \\
\text { (Putaway List) (F2) }\end{array}$} & $\mathrm{n} 1=140$ productos/lista de acomodo \\
\hline & n2=240 productos/lista de acomodo \\
\hline \multirow{2}{*}{ Ubicación del $\operatorname{depot}(\mathrm{F} 3)$} & n1: Centro \\
\hline & n2: Extremo izquierdo del CEDI \\
\hline \multirow{3}{*}{$\begin{array}{l}\text { Grupos de } K \text { EMMateriales } \\
\text { homogéneos (F4). }\end{array}$} & n1: 1 EMMateriales homogéneos (Order Picker) $(K=1)$ \\
\hline & n2: 3 EMMateriales homogéneos ( $K=3$ ) ( 3 Order Picker) \\
\hline & n3: $5(K=5)\left(5\right.$ OrderPicker $\left.{ }^{\star}\right)$ \\
\hline
\end{tabular}


Tabla 5: Análisis de varianza para problema de conformación de lotes en el acomodo

\begin{tabular}{|l|r|r|r|r|r|}
\hline Fuente de variación & SC & gl & CM & F & valor $p$ \\
\hline Modelo. & 35619,14 & 17 & 2095 & 152 & $<0,0001$ \\
\hline GKEquipos & 6748,12 & 2 & 3374 & \multicolumn{2}{|c|}{ Error parcela } \\
\hline GKEquipos>Rep & 0 & 3 & 0 & 0 & $>0,99$ \\
\hline ECL & 12847,84 & 1 & 12848 & 931,02 & $<0,0001$ \\
\hline Depot & 1,26 & 1 & 1,16 & 0,09 & 0,766 \\
\hline TLA comodo & 8771,24 & 1 & 8771,24 & 635,61 & $<0,0001$ \\
\hline ECL*Depot & 0,07 & 1 & 0,07 & 0,01 & $<0,0001$ \\
\hline ECL*TLAcomodo & 748,76 & 1 & 748,76 & 54,26 & 0,973 \\
\hline ECL*GKEquipos & 6140,37 & 2 & 3070,19 & 222,48 & 0,0001 \\
\hline Depot*TLAcomodo & 0,11 & 1 & 0,11 & 0,01 & 0,94 \\
\hline Depot*GKEquipos & 0,73 & 2 & 0,37 & 0,03 & $<0,0001$ \\
\hline TLAcomodo*GKEquipos & 360,64 & 2 & 180 & 13,8 & 0,9292 \\
\hline Error & 413,99 & 30 & 14 & & \\
\hline Total & 36033,14 & 47 & & & \\
\hline
\end{tabular}

En la Tabla 5, se observa que el factor Estrategia de Conformación de Lotes (ECL) de Acomodo y sus interacciones con el tamaño de lista de acomodo (TL acomodo) y el grupo de K Emmateriales son significativos sobre el tiempo promedio de la operación, ya que sus valores p son menores a 0.05 (criterio de rechazo). A partir de estos resultados, se identifica que las interacciones significativas del factor ECL son claves en el diseño o configuración de acomodo buscando obtener el menor tiempo promedio de operación. Además, debe resaltarse que el $R^{2}$ adjustado para el modelo estadístico fue $98 \%$ y las pruebas de normalidad y varianza constante fueron verificadas. Así se garantiza que el modelo estadístico permite realizar un análisis estadístico valido. Del análisis del grafico de medias de la operación (Fig. 4) se identifica que la combinación de niveles Metaheuristico INS con un grupo de 5 EMMateriales genera el menor de acomodo con un tiempo de 18,59 minutos/lotes.

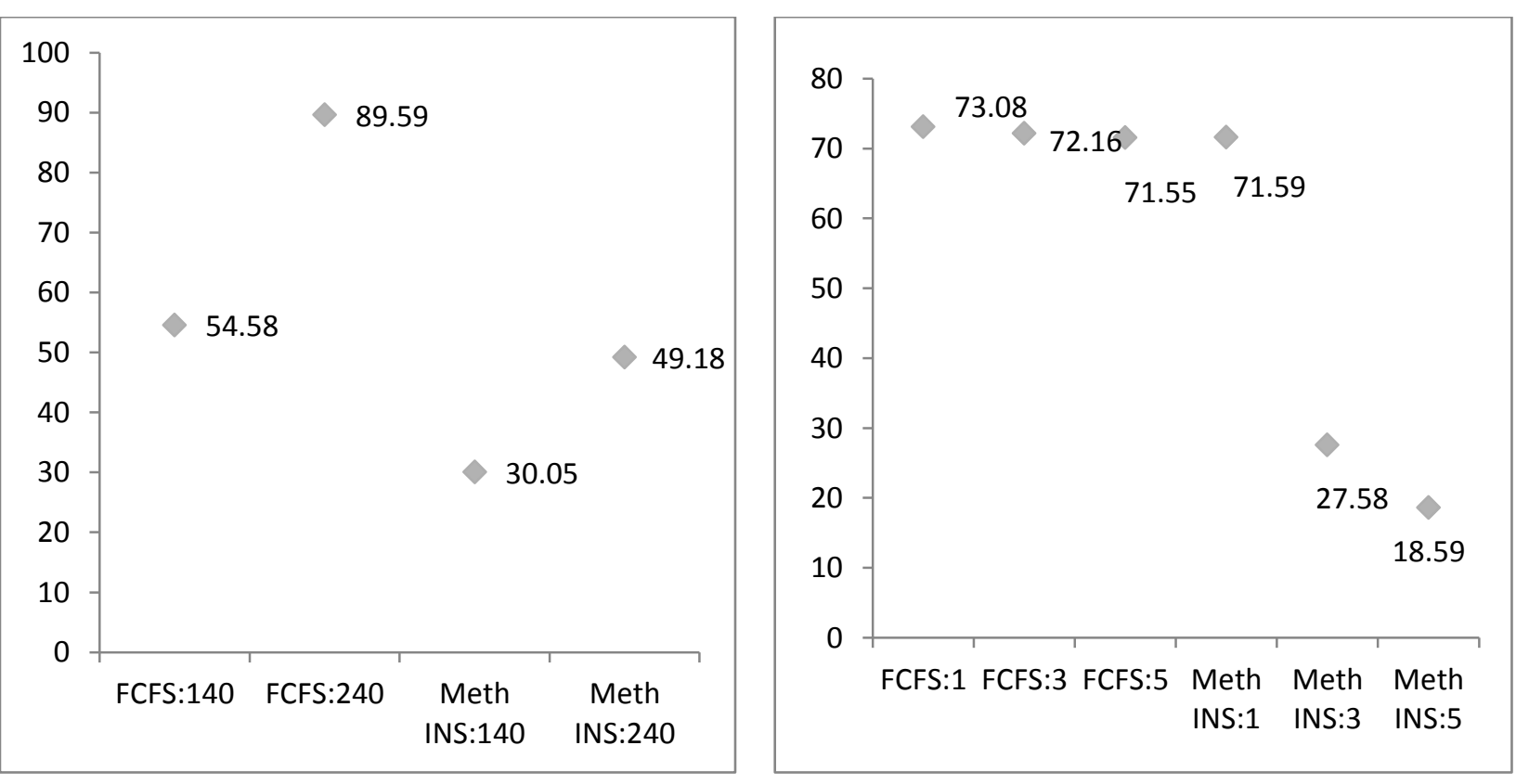

Fig. 4: Tiempo promedio de acomodo para las interacciones dobles de ECL con tamaño de lista de acomodo (TL acomodo) y el grupo de $\mathrm{K}$ Emmateriales. 
Debe resaltarse que para la combinación del metaheurístico INS con un grupo de 3 EMMateriales se obtiene un tiempo promedio de 27,58 minutos/lote. Por este motivo, debería complementarse este análisis de tiempos con uno de costo que determine si un ahorro de 9 minutos/lote justifica el uso de 2 EMMateriales adicionales en la operación de acomodo. Adicionalmente, del análisis de los tiempos promedio de acomodo, se detecta que las combinaciones del metaheurístico INS producen ahorros hasta de 50 minutos/lote según la cantidad de $K$ EMMateriales programados por grupo. Esto resultado evidencia que el metaheurístico INS contribuye a la eficiencia de la operación de acomodo en un almacén de producto terminado.

De otra parte, la mejor interacción doble entre los factores ECL y TLA comodo fueron las combinaciones de Ios niveles metaheurístico INS, los cuales generaron tiempos de 30,05 y 54,58 min/lote para los TLAcomodo de 140 y 240 productos respectivamente. Estos resultados muestran que la ECL metaheurístico INS permiten un ahorro de tiempo de 24 y $40 \mathrm{~min} /$ lote de acomodo respecto a la regla FCFS. De esta manera, se demuestra estadísticamente la reducción en el tiempo promedio de acomodo que produce el metaheurístico INS.

\section{CONCLUSIONES}

Del desarrollo del artículo, se identifica que la conformación de lotes en el acomodo no solo impacta en la eficiencia de la gestión del almacén de producto terminado sino también en la asignación de sus recursos como personal, sistema de almacenamiento y equipo de manejo de materiales. Adicionalmente, con el metaheurístico INS (Intelligent Neighborhood Search) se desarrolla uno de las primeras estrategias de conformación de lotes publicadas en la literatura científica para la operación de acomodo con un enfoque cuantitativo basado en la exploración inteligente de vecindarios.

A partir de los resultados de la experimentación estadística se validó que el metaheurístico INS produjo ahorros de tiempo entre 24 y $50 \mathrm{~min} /$ lote respecto a la regla FCFS en la operación de acomodo utilizada como referencia para el estudio experimentación. Esta reducción de tiempo se justifica porque el metaheurístico INS realiza búsquedas inteligentes que permiten conformar lotes del mínimo tiempo posible lo cual impacta en la eficiencia como fue demostrado. De esta manera, se cumple con el objetivo del artículo que tenía como propósito desarrollar un metaheurístico que resolviera el problema de conformación de lotes en el acomodo considerando $K$ equipos de manejo de materiales (EMMateriales).

Finalmente, como trabajo futuro se plantea modelar grupos de $\mathrm{K}$ EMMateriales homogéneos es decir con diferentes velocidades, capacidades de carga y niveles de altura para ejecutar operaciones de acomodo. Adicionalmente, se desarrollaran variantes al metaheurístico INS planteado respecto a estrategias de exploración de vecindarios así como la integración con metaheurísticos de ruteo con el fin de obtener los menores tiempos posibles de la operación.

\section{REFERENCIAS}

Bartholdi, J. J. y Hackman, S. T., Warehouse \& Distribution Science: Release 0.96. The Supply Chain and Logistics Institute (2014)

Díaz ,E., y Cadena, J., Decisiones fundamentales para estudiar el proceso de alistamiento de pedidos: revisión de literatura. Revista Gerencia Tecnológica Informática, 12(34), 17-28 (2013)

Frazelle, E., y Sojo, R., Logística de Almacenamiento y Manejo de Materiales de Clase Mundial. Grupo Editorial Norma. Bogotá, Colombia (2006)

Henn, S., Algorithms for on-line order batching in an order picking warehouse. Computers \& Operations Research, 39(11), 2549-2563 (2012)

Henn, S., \& Schmid, V., Metaheuristics for order batching and sequencing in manual order picking systems. Computers \& Industrial Engineering, 66(2), 338-351 (2013)

Heragu, S. S., Du, L., Mantel, R. J., y Schuur, P. C., Mathematical model for warehouse design and product allocation. International Journal of Production Research, 43(2), 327-335 (2005)

Hou, J.-L., Wu, Y.-J., y Yang, Y.J., A model for storage arrangement and re-allocation for storage management operations. International Journal of Computer Integrated Manufacturing, 23(4), 369-390 (2010) 
Kim, B. S., y Smith, J. S., Slotting methodology using correlated improvement for a zone-based carton picking distribution system. Computers \& Industrial Engineering, 62(1), 286-295 (2012)

Kulak, O., Sahin, Y., \& Taner, M. E. (2012). Joint order batching and picker routing in single and multiplecross-aisle warehouses using cluster-based tabu search algorithms. Flexible Services and Manufacturing Journal, 24(1), 52-80 (2012)

Kutzelnigg, R. Optimal allocation of goods in a warehouse: Minimizing the order picking costs under real-life constraints. Logistics and Industrial Informatics (LINDI) and 2011 3rd IEEE International Symposium, Budapest, Hungria 25 a 27 de Agosto (2011)

Leung, S. C., Zhang, Z., Zhang, D., Hua, X., \& Lim, M. K., A meta-heuristic algorithm for heterogeneous fleet vehicle routing problems with two-dimensional loading constraints. European Journal of Operational Research, 225(2), 199-210 (2013)

Muppani (Muppant), V. R., y Adil, G. K., Efficient formation of storage classes for warehouse storage location assignment: A simulated annealing approach. Omega, 36(4), 609-618 (2008)

Pazour J. A. y H. J. Carlo. H., Warehouse reshuffling: Insights and optimization», Transportation Research Part E: Logistics and Transportation Review, 73 (1), 207-226 (2015)

Prins, C., Two memetic algorithms for heterogeneous fleet vehicle routing problems. Engineering Applications of Artificial Intelligence, 22(6), 916-928 (2009)

Takahama, H., Nishi, T., Konishi, M., y Imai, J., A determination method of product allocation schedule for warehouse management. En SICE 2002.Proceedings of the 41st SICE Annual Conference (Vol. 2, pp. 10041007 vol.2) (2002)

Theys, C., Bräysy, O., Dullaert, W., \& Raa, B,. Using a TSP Heuristic for routing order pickers in warehouses. European Journal of Operational Research, 200(3), 755-763 (2010)

Van den Berg J. P., Integral Warehouse Management. The Netherlands: Lulu.com. (2007)

Yu, M., \& De Koster, R,. The impact of order batching and picking area zoning on order picking system performance. European Journal of Operational Research, 198(2), 480-490 (2009) 